\title{
Linking CFTR modulators to opportunistic bacterial infections in cystic fibrosis
}

Cristina Cigana ${ }^{1,4}$, Ruggero Giannella ${ }^{1}$, Alice Colavolpe ${ }^{1}$, Beatriz Alcalá-Franco ${ }^{1}$, Giulia Mancini ${ }^{1}$, Colombi Francesca ${ }^{1}$, Chiara Bigogno ${ }^{2}$, Ulla Bastrup ${ }^{2}$, Giovanni Bertoni ${ }^{3}$, Alessandra Bragonzi ${ }^{1,4}$

1 Infections and Cystic Fibrosis Unit, Division of Immunology, Transplantation and Infectious Diseases, IRCCS San Raffaele Scientific Institute, Milan, Italy

${ }^{2}$ Aphad Srl, Buccinasco, Italy

${ }^{3}$ Department of Biosciences, Università degli Studi di Milano, Milan, Italy

${ }^{4}$ Both authors share senior authorship

Corresponding authors: Infections and Cystic Fibrosis Unit, Division of Immunology, Transplantation and Infectious Diseases, IRCCS San Raffaele Scientific Institute, via Olgettina 58, 20132 Milano, Italy (cigana.cristina@hsr.it and alessandra.bragonzi@hsr.it)

Running title: CFTR modulators and bacterial infections

Keywords: cystic fibrosis, CFTR modulator, Pseudomonas aeruginosa, Staphylococcus aureus, antibiotic, murine model, pharmacokinetics

Manuscript number of words: 3495

Manuscript number of figures and tables: 7 


\section{Abstract}

2 Cystic fibrosis transmembrane conductance regulator (CFTR) modulators improve clinical outcomes

3 with variable efficacy in patients with cystic fibrosis (CF). However, changes produced by bacterial

4 persistence and adaptation in addition to antibiotic regimens could influence CFTR modulator 5 efficacy and vice versa and hence clinical outcomes. We first evaluated the effects of ivacaftor (IVA), lumacaftor (LUM), tezacaftor, elexacaftor and elexacaftor/tezacaftor/ivacaftor (ETI), alone or combined with antibiotics, on sequential Staphylococcus aureus and Pseudomonas aeruginosa CF isolates. IVA and ETI showed the most potent direct antimicrobial activity against $S$. aureus, while $P$. aeruginosa was not affected. Additive effects or synergies were observed between the CFTR modulators and antibiotics against both $S$. aureus and $P$. aeruginosa, independently of the stage of colonization. IVA and LUM were the most effective in potentiating antibiotic activity against $S$. aureus, while IVA and ETI enhanced mainly polymyxins activity against $P$. aeruginosa. Next, we evaluated the effect of $P$. aeruginosa pneumonia on the pharmacokinetics of IVA in mice. The timeconcentration curves of IVA and its metabolites in plasma, lung and epithelial lining fluid were influenced by $P$. aeruginosa infection. The area under the concentration-time curve showed that airway exposure to IVA was greater in infected than non-infected mice. These results suggest that CFTR modulators can have direct antimicrobial properties and/or enhance antibiotic activity against $S$. aureus and $P$. aeruginosa. Furthermore, bacterial infection impacts the IVA concentration and airway exposure, potentially affecting its efficacy. Our findings suggest optimizing host- and pathogen-directed drug regimens to improve efficacy for personalized treatment. 


\section{Introduction}

Cystic fibrosis (CF) is caused by mutations in the cystic fibrosis transmembrane conductance regulator (CFTR) gene and affects mostly the lung, but also other organs. Respiratory failure is caused by pulmonary infections and inflammation [1], with Pseudomonas aeruginosa and Staphylococcus aureus being recognized as the most prevalent pathogens. Their chronic persistence and pathogenicity are associated with adaptation to the CF airways [2].

Traditionally, treatments focused on lessening infection and inflammation of CF disease, although efficacy is not optimal. Recently, new approaches to correct CFTR cellular misprocessing (correctors) and restore its channel function (potentiators) have emerged [3]. The CFTR potentiator ivacaftor (IVA) was introduced to target gating and other residual function deficits [4-6], while the CFTR correctors lumacaftor (LUM), tezacaftor (TEZ) and elexacaftor (ELX) were approved to correct the F508del-CFTR protein and recently extended to other mutations causing processing and trafficking defects. Since the commonest F508del-CFTR requires both correction and potentiation for clinical efficacy, two dual- (LUM/IVA and TEZ/IVA) [7-11] and triple- (ELX/TEZ/IVA named ETI) [12] agent drugs were approved. Despite the clinical benefits of these treatments, marked variability in CF patients has been reported $[4,5,7,13]$. This supports the view that the response to CFTRdirected therapeutics is multifactorial and that CFTR-independent factors may contribute to treatment efficacy, but the mechanisms remain unknown.

There continues to be a substantial gap in our understanding of whether and how CFTR-directed therapies impact the microbiological profile in treated patients [14, 15]. IVA treatment showed an initial reduction in the prevalence of bacterial isolation, but $P$. aeruginosa density rebounded after one year [16], and $P$. aeruginosa strains present before treatment persisted even after intensive antibiotic therapy [17]. In vitro studies have shown the anti-S. aureus activity of IVA and its synergy with anti-P. aeruginosa antibiotics [18-23]. LUM and TEZ have also been shown to enhance the activity of polymyxin B (PMB) against some $P$. aeruginosa isolates [21], while the impact of ELX and ETI remains largely unexplored. Whether the activity of CFTR modulators differs in CF strains associated with early and advanced stages of lung colonization remains unclear.

To monitor possible interactions between CFTR modulators and CF pathogens, it is critical to know the drug concentration in the lung, particularly in the epithelial lining fluid (ELF), where bacteria are present [24]. Most pharmacokinetic (PK) studies have evaluated the amount of CFTR modulators and their metabolites in the plasma of different hosts [25, 26], while few data on human sputum have been reported [27]. More importantly, the amounts of CFTR modulators that reach the airway lumen and whether bacterial pneumonia can alter their PK profiles are unclear. Considering that most CF patients are likely colonized by bacteria when CFTR modulator therapy is initiated [14], there is a clinical need to assess the impact of infection on drug metabolism to determine the correct dosage. Although mouse models are not helpful for efficacy studies, they can facilitate PK analysis and provide much-needed knowledge on the interactions between CFTR modulators and CF pathogens avoiding variables of human studies.

Thus, we aimed to establish whether and how the bacterial adaptation affects the antimicrobial activity of CFTR modulators, including ELX and ETI, and the impact of bacterial infection on PK. We designed the study to i) determine the antimicrobial activity of CFTR modulators and their additive or synergistic effects with antibiotics against $P$. aeruginosa and $S$. aureus reference and clonal isolates collected from CF patients at early and advanced stages of lung colonization; ii) evaluate the concentration of IVA, as a model CFTR modulator, in plasma and airway samples and its changes during pneumonia in mice.

\section{Methods}

Ethics statement. The animal studies adhered to the Italian Ministry of Health guidelines for the use and care of experimental animals (IACUC \#954). The experiments with CF P. aeruginosa and S. aureus isolates and storage of biological materials were approved by the Ethics Commissions of Hannover Medical School and University Hospital Münster (Germany). 
Bacterial strains. $P$. aeruginosa and $S$. aureus strains included reference PAO1 and Newman. CF clinical isolates recovered at early and late chronic colonization were RP73, AA2, AA43, AA44, MF1, MF51, KK1, KK2, KK71 and KK72 for $P$. aeruginosa and A10, A12, J6, J9, F1 and F5 [28-31] for S. aureus. Phenotype and antimicrobial resistance were previously characterized (Supplementary Tables S1 and S2) [28-31].

Minimum inhibitory concentration (MIC) measurement. The MICs of CFTR modulators were determined using the broth microdilution susceptibility testing method as described in Supplementary Methods [28, 32].

Checkerboard assay. The synergistic activities of the CFTR modulators combined with tobramycin (TOB), ciprofloxacin (CIP), colistin (CST), PMB, meropenem (MER) and azithromycin (AZM) for $P$. aeruginosa and amoxicillin (AMX), teicoplanin (TEC), linezolid (LZD), vancomycin (VAN) and AZM for $S$. aureus, were determined by the checkerboard method in cation-adjusted Mueller-Hinton broth [33], as detailed in the Supplementary Methods.

Mouse model. C57BL/6NCrIBR male mice (8-10 weeks of age) were challenged with $1 \times 10^{6}$ colony forming units of the planktonic PAO1 by intratracheal injection. Mice were treated with $3 \mathrm{mg} / \mathrm{kg}$ IVA or vehicle (10\% PEG $400,10 \%$ Tween 80 , and $80 \%$ saline) [19] by intraperitoneal injection half an hour after infection, according to the ARRIVE guidelines [34]. IVA dose was calculated based on a single adult dose $(150 \mathrm{mg})$ adjusted for mouse weight, assuming that an adult with CF weighs $50 \mathrm{~kg}$ [19]. Blood, bronchoalveolar lavage fluid (BALF) and lung samples were recovered at different time points, as described [35] for PK and reported in Supplementary Methods.

Protein binding and concentration. Protein binding was measured in mouse plasma and lung homogenate by Rapid Equilibrium Dialysis. Samples were analysed by ultra-performance liquid chromatography-tandem mass spectrometry. Quantitation was performed via multiple reaction monitoring using the transitions reported in Supplementary Table S3. Concentrations of IVA and its metabolites in ELF were determined by using the ratio of the urea concentration in BALF to that in plasma [36]. Protein binding is expected to be negligible in ELF [36]. The drug concentration in ELF was assumed to be equal to the unbound concentration. Additional details are reported in the Supplementary Methods.

PK evaluation. PK parameters were estimated using PK Solver Excel using a non-compartmental approach consistent with the intraperitoneal route of IVA administration. The area under the concentration-versus-time curve was calculated using the linear trapezoidal method for the period from $t=0$ to the time of the last quantifiable concentration level $\left(A \cup C_{0-\text { last }}\right)$. Evaluation of the terminal elimination phase was not practical, as terminal phase concentration data were either not available (low doses) or sparse.

Statistics. Statistical analyses were performed with GraphPad Prism (GraphPad Software, Inc., San Diego, CA, USA) using a non-parametric two-tailed Mann-Whitney $U$ test. A value of $p \leq 0.05$ was considered statistically significant.

\section{Results}

IVA and ETI show the most potent antimicrobial activity against longitudinal $S$. aureus isolates, while $P$. aeruginosa isolates are not affected

To evaluate the impact of the bacterial adaptation on the antimicrobial activity of CFTR modulators we measured MICs against clinical clonal isolates, collected from CF patients at early and late stage of chronic colonization (Supplementary Tables S1 and S2) [28-31]. Single CFTR modulators and the ELX/TEZ/IVA combination at a ratio of 2/1/1.5, which is used for CF patients [37], were tested. IVA showed antimicrobial activity with MICs ranging from 2 to $8 \mu \mathrm{g} / \mathrm{ml}$ for all the $S$. aureus isolates (Table 1). The MIC of ELX was $16 \mu \mathrm{g} / \mathrm{ml}$ for almost all $S$. aureus isolates, except for the $\mathrm{J} 6$ isolate $(32 \mu \mathrm{g} / \mathrm{ml})$. By contrast, the MICs of LUM and TEZ were $>32 \mu \mathrm{g} / \mathrm{ml}$, indicating little or no effect against any of the $S$. aureus isolates. The ELX/TEZ/IVA combination showed antimicrobial activity 
against all the $S$. aureus isolates, with MICs ranging from 4/2/3 to $8 / 4 / 6 \mu \mathrm{g} / \mathrm{ml}$. By contrast, all CFTR modulators showed MICs $>32 \mu \mathrm{g} / \mathrm{ml}$ against $P$. aeruginosa isolates (Supplementary Table S4). Our results demonstrate that adaptation does not change either the susceptibility of $S$. aureus strains to IVA and ETI or the resistance of $P$. aeruginosa to CFTR modulators.

\section{IVA and LUM potentiate the activity of all antibiotics against $S$. aureus}

Next, we determined potential additive or synergistic effects of the CFTR modulators in combination with antibiotics against reference and clinical $S$. aureus isolates by checkerboard assays. In general, except for TEZ, interaction effects were observed on some isolates and regardless of the stage of colonization (Table 2). The fractional inhibitory concentration (FIC) indexes of IVA showed broadspectrum potentiation of LZD activity ( 6 out of 7 isolates), while a narrower spectrum was observed for AMX, VAN, and TEC. Synergistic effects were observed also between IVA and either VAN or TEC but only in the Newman reference strain. By contrast, additive effects between either ELX or ETI and the tested antibiotics were more sporadic or completely absent, as in the case of LZD. LUM, which did not show direct antibiotic effects, behaved similarly to IVA in elevating the activities of the tested antibiotics and showed better synergistic effects with VAN or TEC against the Newman strain. These findings indicate that the CFTR modulators, mainly IVA and LUM, can enhance the performance of antibiotics used against $S$. aureus infections in CF patients. The potentiating effects appear to be bacterial isolate dependent, but no correlation with the stage of chronic colonization was found.

\section{IVA and ETI potentiate the activity of only selected antibiotics against $P$. aeruginosa}

Despite the lack of intrinsic antibacterial activity of the CFTR modulators against $P$. aeruginosa, we tested whether they enhance the activity of antibiotics (Table 3). Strikingly, IVA was found to strongly enhance the activity of CST and PMB against $95.45 \%$ of the isolates. Synergy, rather than additivity, was detected in most of the isolates. No effect was observed when IVA was combined with CIP, MER or TOB. LUM, TEZ and ELX showed only a few cases of additive or synergistic effects with selected antibiotics. For ETI, the FIC values indicated an additive effect on the activities of CST and $\mathrm{PMB}$ in several isolates, with synergy against the AA2 isolate reached with both antibiotics. In only two isolates, ETI potentiated the activity of CIP. No CFTR modulators affected AZM activity on $P$. aeruginosa isolates. Notably, no negative interaction of the CFTR modulators with the antibiotics was observed. These findings indicate that IVA and ETI can enhance antibiotic activity, particularly that of CST and PMB, against $P$. aeruginosa in an isolate-dependent manner and independently of the stage of colonization.

\section{IVA is differentially distributed in plasma, lung and ELF, with concentrations affected by $P$. aeruginosa infection}

To establish the impact of bacterial infection on CFTR modulators PK, we exploited the mouse model of $P$. aeruginosa pneumonia [35] and evaluated IVA concentration in murine plasma, lung and ELF. We selected IVA as the CFTR modulator with the most striking anti-microbial activity and $P$. aeruginosa as the most relevant CF pathogen [38]. We first determined the protein binding of IVA and its metabolites M1 (pharmacologically active) and M6 (inactive) [39]. The percentages of protein binding were very high in the plasma, with those of IVA, M1 and M6 being $\geq 99.5 \%$ (Table 4). Similar protein binding was observed in the lung for IVA $(99.7 \%)$ and M1 $(97.2 \%)$ while that of M6 was lower $(80.3 \%)$.

Next, the time-concentration curves of IVA and its metabolites showed that parent drug accounted for the majority of the total drug, followed by $M 1$ and then M6, in the plasma of both infected and non-infected mice (Figure 1A). At early time points, IVA and M1 levels were lower in $P$. aeruginosainfected compared to non-infected mice $(p=0.057)$. However, at later time points, the IVA levels were slightly higher in infected compared to non-infected mice. M6 levels ranged from low to undetectable in the plasma of all mice. Peak plasma levels of IVA, reached $1 \mathrm{hr}$ after treatment, were higher in non-infected compared to infected mice (Table 5), as were M1 levels (Supplementary Table S5). Nonetheless, the AUC for the period from $t_{10 \text { min }}$ to the last quantifiable concentration $\left(A U C_{\text {last }}\right)$ and the $A \cup C$ to infinity $\left(A \cup C_{\text {inf }}\right)$ for IVA were higher in $P$. aeruginosa-infected than non-infected mice, 
indicating higher exposure in the presence of infection. This was confirmed by higher last quantifiable concentration ( $\mathrm{C}_{\text {last }}$ ) at $24 \mathrm{hrs}$, half-life $\left(\mathrm{T}_{1 / 2}\right)$ and mean residence time (MRT) in infected compared to non-infected mice. By contrast, M1 AUCs, $\mathrm{C}_{\text {last, }} \mathrm{T}_{1 / 2}$ and MRT were similar in both groups.

The IVA profile in the ELF followed that in the plasma samples up to $6 \mathrm{hrs}$, with higher levels in noninfected compared to infected mice soon after treatment $(p=0.057$; Figure 1B). P. aeruginosa infection resulted in higher IVA peak levels, $A U C_{\text {last }}, A U C_{\text {inf }}$ and $C_{\text {last }}$ compared to non-infected mice (Table 5). The partitioning to the ELF was estimated by comparing the AUC of the unbound compound (fAUC) in the plasma to that in the ELF. For the calculation of fAUC, a factor of $0.03 \%$ was used for the IVA parent drug in the plasma (Table 4). The ELF/plasma fAUC ratio of IVA was high, indicating that IVA penetrates ELF well (Table 6). M1 was only detected between 1 and 6 hrs post-administration in ELF of infected and non-infected mice (Figure 1B). The ELF/plasma fAUC ratio was also high for $M 1$, indicating high penetration in the ELF. M6 was not quantifiable for any conditions.

The IVA profile in the lung homogenates showed similar levels in infected and non-infected mice up to $2 \mathrm{hrs}$, while the levels were higher in $P$. aeruginosa-infected mice after $6 \mathrm{hrs}$ (Supplementary Figure S1). The lung/plasma fAUC ratio of IVA indicated that IVA penetrates the lung very well, particularly in infected mice (Table 6). M1 showed a profile in the lung similar to that in the ELF in both infected and non-infected mice (Supplementary Figure S1 and Supplementary Tables S5 and S6). M6 was not quantifiable in either infected or non-infected mice at any time point.

These findings indicated that the IVA parent drug, accounting for the majority of the total drug, readily distributes to the airways and is higher during $P$. aeruginosa infection.

\section{Discussion}

CFTR modulators correct the molecular defect underlying CF and disease manifestations. Since bacterial lung infections/colonization are one of the hallmarks of CF disease, the effect (if any) of CFTR modulators on bacteria could deeply affect the course of the disease. Here, we tested the impact of bacterial adaptation on the antimicrobial activity of CFTR modulators and that of bacterial infection on PK. We found that: 1) IVA and newly licensed ETI have the most potent intrinsic antimicrobial activity against longitudinal $S$. aureus isolates, while $P$. aeruginosa isolates are not affected; 2) IVA and LUM potentiate the activity of all antibiotics against $S$. aureus, while IVA and ETI especially that of polymyxins against $P$. aeruginosa; 3 ) antimicrobial activity of CFTR modulators is independent of the stage of colonization for both bacterial species; 4$) P$. aeruginosa infection affects the distribution of IVA to plasma, lung and ELF.

Our study showed that IVA has a spectrum of relevant antibacterial activity that spans both early and late isolates of $S$. aureus. In addition, ETI is active against $S$. aureus and its activity seems to be mainly driven by IVA since the effective concentration of IVA in the triple combination was in the range of that as a single drug. In addition, we showed for the first time that ELX has broad antibacterial activity against $S$. aureus. Notably, the anti-S. aureus activity of CFTR modulators is independent of the stage of colonization. These activities deserve further investigation with additional biobanks, including those from CF patients under CFTR modulators treatment. Regarding CFTR modulator and antibiotic interactions, we expanded the notion that IVA can increase the activity of several classes of antibiotics against $S$. aureus. Furthermore, we show that LUM, with no intrinsic antibacterial activity against $S$. aureus, can positively interact with several classes of antibiotics. The specific mechanism underlying the enhancement of antibiotics activity by IVA and LUM against $S$. aureus is unknown and should be investigated.

We observed a different scenario for $P$. aeruginosa. IVA showed no antibacterial activity against any $P$. aeruginosa early and late isolates, suggesting that adaptation to the CF environment does not induce modification of bacterial structures or functions potentially causing resistance to IVA. This is in line with other reports showing that IVA is inactive against $P$. aeruginosa and other gram-negative species (e.g. Klebsiella pneumoniae and Acinetobacter baumannii) [21]. Gram-negative bacteria have a formidable outer membrane barrier, particularly against hydrophobic drugs as CFTR modulators, that can explain the absence of IVA activity. Alternatively, IVA might not affect essential 
$P$. aeruginosa pathways and can be effective only as a combined treatment. Indeed, we observed that IVA can specifically act in concert with polymyxins, such as CST and PMB, to enhance their antibacterial activity in almost all the isolates tested, while no additive or synergistic effects were observed for the other classes of antibiotics. Recently, IVA in combination with PMB has been shown to potentiate bactericidal activity against $P$. aeruginosa by inhibiting cell envelope biogenesis [23]. Similar to IVA, also ETI potentiated the activities of CST and PMB in several isolates. Although the activity of ETI can be driven by IVA, some exceptions have been observed, suggesting potential antagonisms when IVA is combined in the triple combination in an isolate-dependent manner.

To further interpret these results, it is critical to know the amounts of CFTR modulators that reach the airway lumen and whether bacterial pneumonia can alter their concentrations. We focused on IVA since this CFTR modulator exhibited the most effective antimicrobial activity independently or in combination with antibiotics. To investigate the PK profile and provide insight on the interactions between CFTR modulators and CF pathogens in the absence of confounding variables of human studies, we exploited a mouse model of $P$. aeruginosa pneumonia. Our results showed that the parent drug accounted for the majority of the total drug in plasma, lung and ELF, similar to previous reports focused solely on murine plasma but different from humans, who show higher concentrations of M1 in plasma [25]. Interestingly, exposure of both plasma and airways to IVA was higher in mice with acute $P$. aeruginosa lung infection than in non-infected mice. In our study, the IVA $C_{\max }$ in the plasma was in the range of a few $\mu \mathrm{g} / \mathrm{ml}$. However, the protein binding of IVA and its metabolites was extremely high in both the plasma and lung, indicating that the amount of free active compound is low, in agreement with previous studies [25]. For instance, the $C_{\max }$ of free active IVA in plasma was in the range of approximately $1 \mathrm{ng} / \mathrm{ml}$ in our model, similarly to the peak free plasma concentration measured in CF patients treated with IVA [26].

When we focused on the airways, IVA showed good penetration into the ELF, reaching higher concentrations than those in the plasma. The IVA $C_{\max }$ was approximately $0.5-1 \mu \mathrm{g} / \mathrm{ml}$ in the ELF. Notably, we observed antimicrobial activity or additive/synergistic effects with CST at IVA concentrations ranging from 1 to $8 \mu \mathrm{g} / \mathrm{ml}$, which were comparable to or higher than those observed in the murine ELF. Schneider and colleagues measured an IVA concentration of $0.15 \pm 0.05 \mu \mathrm{g} / \mathrm{ml}$ in the sputum of a CF patient at $2.5 \mathrm{hrs}$ post IVA administration [27], which would exclude antimicrobial activities of CFTR modulators in CF patients. However, data from sputa of single patients cannot be considered conclusive, and IVA quantification in a cohort of patients is needed. In addition, IVA is known to accumulate with repeated doses, particularly when it is taken with a fatty meal [40]. Our work analysed IVA concentrations in mice after administering a single dose, but higher levels are expected during chronic treatment. In this regard, IVA levels following prolonged treatment in CF patients are unknown. Therefore, the antimicrobial activities of IVA in the airways of CF patients could be plausible and deserves further investigation. In addition our findings support performing new studies on the potential benefits of pulmonary administration of CST in combination with IVA in treating $P$. aeruginosa lung infections [41].

\section{Conclusions}

This work supports the interaction of CFTR-modulators with opportunistic bacterial infection and antibiotics. Our results underline that CFTR-modulators influence antibiotic efficacy through a direct or synergistic effect. This may orient the selection of specific antibiotics to treat infection in combination with CFTR-modulators. Importantly, CFTR-modulator efficacy is not influenced by specific bacterial phenotypes (early vs late adapted), indicating drug targeting at any stage of colonization. Since the bacterial strains used in this study were collected several years ago, they do not show some of the antibiotic resistances that have emerged in CF clinics in recent years. The challenge ahead is to validate our results with additional biobanks that include longitudinal strains isolated from CF patients under CFTR modulator therapies and with different patterns of antibiotic resistance.

So far, both animal and human studies did not clarify the impact of infection on concentrations and biodistribution of CFTR modulators. Our results in a mouse model of $P$. aeruginosa pneumonia underline the importance of testing PK during infection, particularly in the airways. This would help to optimize the drug regimens improving the efficacy in the view of personalized treatment. 


\section{Acknowledgements}

284 We thank Marzia Giustra for her invaluable contribution to the in vitro assays, and Burkhard Tümmler (Medizinische Hochschule Hannover, Germany) and Barbara C. Kahl (University Hospital Münster, Germany) for supplying respectively $P$. aeruginosa and $S$. aureus clinical isolates collected from CF patients. This study was supported to CC by the Italian Cystic Fibrosis Research Foundation (FFC\#15/2018), with the contribution of the Delegazione FFC di Milano and Gruppo di Sostegno FFC di Morbegno, and to AB by the Cystic Fibrosis Foundation (BRAGON19G0). The funders had no role in study design, data collection and analysis, decision to publish, or preparation of the manuscript.

\section{Author contribution}

$\mathrm{CC}$ and BA contributed to the conception and design of the study. GR, CA and MG performed MIC and checkerboard assays. CC, AFB and CF performed in vivo experiments and collected data. BC and $\mathrm{BU}$ evaluated protein binding, and compound quantification in murine samples. $\mathrm{CC}$ analyzed data and performed the statistical analysis. $\mathrm{CC}$ and $\mathrm{BC}$ prepared the figures and tables. CC wrote the first draft of the manuscript. BG and BA critically revised the manuscript. All the authors read, revised and approved the final manuscript. 
Table 1. Minimum inhibitory concentrations (MIC ${ }_{90}$ ) of ivacaftor (IVA), lumacaftor (LUM), tezacaftor (TEZ), elexacaftor (ELX) and the triple combination elexacaftor/tezacaftor/ivacaftor (ELX/TEZ/IVA) against $S$. aureus isolates collected from CF patients at different stages of colonization (early and late). $S$. aureus isolates were grown for $20 \mathrm{hrs}$ in the presence of serial dilutions of IVA, LUM, TEZ, ELX and the triple combination ELX/TEZ/IVA. The concentrations tested ranged from $0.25 \mu \mathrm{g} / \mathrm{ml}$ to $32 \mu \mathrm{g} / \mathrm{ml}$ for IVA, LUM, TEZ and ELX and from ELX $0.5 \mu \mathrm{g} / \mathrm{ml} / \mathrm{TEZ} 0.25$ $\mu \mathrm{g} / \mathrm{ml} / \mathrm{IVA} 0.375 \mu \mathrm{g} / \mathrm{ml}$ to ELX $64 \mu \mathrm{g} / \mathrm{ml} / \mathrm{TEZ} 32 \mu \mathrm{g} / \mathrm{ml} / \mathrm{IVA} 48 \mu \mathrm{g} / \mathrm{ml}$ for the triple combination. The $\mathrm{MIC}_{90}$ was defined as the lowest compound concentration showing a reduction in the optical density at $620 \mathrm{~nm}$ of approximately $90 \%$ in comparison to the optical density of the bacteria grown with the vehicle after 20 hrs. Each experiment was performed at least two independent times (two technical replicates).

314

315

316

\section{S. aureus isolates}

\begin{tabular}{|c|c|c|c|c|c|c|c|c|}
\hline \multicolumn{2}{|c|}{ Isolate name } & Newman & A10 & A12 & J6 & J9 & F1 & F5 \\
\hline \multicolumn{2}{|c|}{ Isolate type } & ref & early & late & early & late & early & late \\
\hline \multirow{5}{*}{$\begin{array}{l}\text { MIC }_{90} \\
(\mu \mathrm{g} / \mathrm{ml})\end{array}$} & IVA & 4 & 2 & 4 & 8 & 8 & 8 & 8 \\
\hline & LUM & $>32$ & $>32$ & $>32$ & $>32$ & $>32$ & $>32$ & $>32$ \\
\hline & TEZ & $>32$ & $>32$ & $>32$ & $>32$ & $>32$ & $>32$ & $>32$ \\
\hline & ELX & 16 & 16 & 16 & 32 & 16 & 16 & 16 \\
\hline & ELX/TEZ/IVA & $4 / 2 / 3$ & $4 / 2 / 3$ & $4 / 2 / 3$ & $8 / 4 / 6$ & $8 / 4 / 6$ & $8 / 4 / 6$ & $4 / 2 / 3$ \\
\hline
\end{tabular}

ref, reference strain; early, isolate collected at the early stage of chronic colonization; late, isolate collected after years of persistence, in the advanced stage of chronic colonization.

Table 2. Fractional inhibitory concentration (FIC) indexes for ivacaftor (IVA), lumacaftor (LUM), tezacaftor (TEZ), elexacaftor (ELX) and the triple combination elexacaftor/tezacaftor/ivacaftor (ELX/TEZ/IVA) in combination with common antibiotics against $S$. aureus isolates collected from CF patients. $S$. aureus isolates were grown for $20 \mathrm{hrs}$ in the presence of serial dilutions of IVA, LUM, TEZ, ELX or the triple combination ELX/TEZ/IVA (ETI) and amoxicillin (AMX), vancomycin (VAN), teicoplanin (TEC), linezolid (LZD), or azithromycin (AZM). The concentrations of the CFTR modulators tested ranged from $0.5 \mu \mathrm{g} / \mathrm{ml}$ to $32 \mu \mathrm{g} / \mathrm{ml}$ for IVA, LUM and ELX and from ELX $0.5 \mu \mathrm{g} / \mathrm{ml} / T E Z ~ 0.25 \mu \mathrm{g} / \mathrm{ml} / \mathrm{IVA} 0.375 \mu \mathrm{g} / \mathrm{ml}$ to ELX $32 \mu \mathrm{g} / \mathrm{ml} / \mathrm{TEZ}$ $16 \mu \mathrm{g} / \mathrm{ml} / \mathrm{IVA} 24 \mu \mathrm{g} / \mathrm{ml}$ for the triple combination ETI, while those of the antibiotics started from the concentration two-fold greater than the $\mathrm{MIC}_{90}$ for the isolate to 32 times less than the $\mathrm{MIC}_{90}$ of the antibiotic alone. FIC indexes, calculated as $\mathrm{MIC}_{90}$ of CFTR modulator in combination/ $\mathrm{MIC}_{90}$ CFTR alone $+\mathrm{MIC}_{90}$ antibiotic in combination/ $\mathrm{MIC}_{90}$ antibiotic alone, are indicated. Each experiment was performed at least two independent times (two technical replicates).

\begin{tabular}{|c|c|c|c|c|c|c|c|c|c|}
\hline & & & \multicolumn{7}{|c|}{$S$. aureus isolates } \\
\hline & & & Newman & A10 & A12 & J6 & J9 & F1 & F5 \\
\hline & & & ref & early & late & early & late & early & late \\
\hline \multirow{2}{*}{ 는 $\stackrel{*}{\stackrel{*}{0}}$} & \multirow{2}{*}{ IVA } & AMX & 0.5 & 1 & 2 & 1 & 0.5 & 0.625 & 0.625 \\
\hline & & VAN & 0.31 & 0.625 & 0.625 & 1 & 1 & 1 & 1 \\
\hline
\end{tabular}




\begin{tabular}{|c|c|c|c|c|c|c|c|c|}
\hline & TEC & 0.31 & 1 & 1 & 1 & 0.75 & 1 & 0.75 \\
\hline & LZD & 0.75 & 2 & 0.625 & 0.625 & 0.625 & 0.625 & 0.625 \\
\hline & AZM & 0.531 & 2 & 2 & 2 & 2 & 2 & 2 \\
\hline \multirow{5}{*}{ LUM } & AMX & 0.625 & 0.625 & 2 & 1 & 0.625 & 0.625 & 0.625 \\
\hline & VAN & 0.155 & 0.625 & 0.75 & 1 & 1 & 1 & 0.625 \\
\hline & TEC & 0.185 & 0.625 & 0.625 & 2 & 2 & 0.625 & 0.75 \\
\hline & LZD & 0.625 & 2 & 0.625 & 2 & 0.75 & 0.625 & 0.625 \\
\hline & AZM & 0.75 & 1 & 1 & 2 & 2 & 1 & 2 \\
\hline \multirow{5}{*}{ TEZ } & AMX & 2 & 2 & 2 & 2 & 2 & 2 & 2 \\
\hline & VAN & 2 & 2 & 2 & 2 & 2 & 2 & 2 \\
\hline & TEC & 2 & 2 & 2 & 2 & 2 & 2 & 2 \\
\hline & LZD & 2 & 2 & 2 & 2 & 2 & 2 & 2 \\
\hline & AZM & 2 & 2 & 2 & 2 & 1 & 2 & 2 \\
\hline \multirow{5}{*}{ ELX } & AMX & 2 & 0.5 & 0.75 & 2 & 0.75 & 2 & 2 \\
\hline & VAN & 2 & 0.563 & 2 & 2 & 2 & 2 & 2 \\
\hline & TEC & 2 & 2 & 2 & 2 & 2 & 2 & 2 \\
\hline & LZD & 2 & 2 & 2 & 2 & 2 & 2 & 2 \\
\hline & AZM & 0.75 & 1 & 1 & 0.75 & 0.75 & 0.5 & 2 \\
\hline \multirow{5}{*}{ ETI } & AMX & 2 & 0.75 & 1 & 2 & 0.75 & 2 & 2 \\
\hline & VAN & 2 & 2 & 2 & 2 & 2 & 2 & 2 \\
\hline & TEC & 2 & 2 & 2 & 0.625 & 2 & 1 & 2 \\
\hline & LZD & 2 & 2 & 2 & 2 & 2 & 2 & 2 \\
\hline & AZM & 2 & 2 & 2 & 2 & 2 & 2 & 2 \\
\hline
\end{tabular}

${ }^{*} \mathrm{FIC}$ index $<0.5$ indicates synergy (highlighted in green), FIC index $>0.50$ and $<1.0$ indicates additive effect (highlighted in yellow), FIC index $>1.0$ and $<4$ indicates indifference. Ref, reference strain; early, isolate collected at the early stage of chronic colonization; late, isolate collected after years of persistence, in the advanced stage of chronic colonization.

Table 3. Fractional inhibitory concentration (FIC) indexes for ivacaftor (IVA), lumacaftor (LUM), tezacaftor (TEZ), elexacaftor (ELX) and the triple combination elexacaftor/tezacaftor/ivacaftor (ELX/TEZ/IVA) in combination with common antibiotics against $\boldsymbol{P}$. aeruginosa isolates collected from CF patients. $P$. aeruginosa isolates were grown for $20 \mathrm{hrs}$ in the presence of serial dilutions of IVA, LUM, TEZ, ELX or the triple combination ELX/TEZ/IVA and ciprofloxacin (CIP), meropenem (MER), colistin (CST), tobramycin (TOB), polymyxin $B(P M B)$ and azithromycin (AZM). The concentrations of the CFTR modulators tested ranged from $0.5 \mu \mathrm{g} / \mathrm{ml}$ to $32 \mu \mathrm{g} / \mathrm{ml}$ for IVA, LUM, TEZ and ELX, and from ELX $0.5 \mu \mathrm{g} / \mathrm{ml} / \mathrm{TEZ} 0.25$ $\mu \mathrm{g} / \mathrm{ml} / \mathrm{IVA} 0.375 \mu \mathrm{g} / \mathrm{ml}$ to ELX $32 \mu \mathrm{g} / \mathrm{ml} / \mathrm{TEZ} 16 \mu \mathrm{g} / \mathrm{ml} / \mathrm{IVA} 24 \mu \mathrm{g} / \mathrm{ml}$ for the triple combination ETI, while those of the antibiotics started from the concentration two-fold greater than the $\mathrm{MIC}_{90}$ for the 
344 isolate to 32 times less than the $\mathrm{MIC}_{90}$ of the antibiotic alone. FIC indexes, calculated as $\mathrm{MIC}_{90}$ of CFTR modulator in combination/ $\mathrm{MIC}_{90} \mathrm{CFTR}$ alone + $\mathrm{MIC}_{90}$ antibiotic in combination/ $\mathrm{MIC}_{90}$ antibiotic alone, are indicated. Each experiment was performed at least two independent times (two technical replicates).

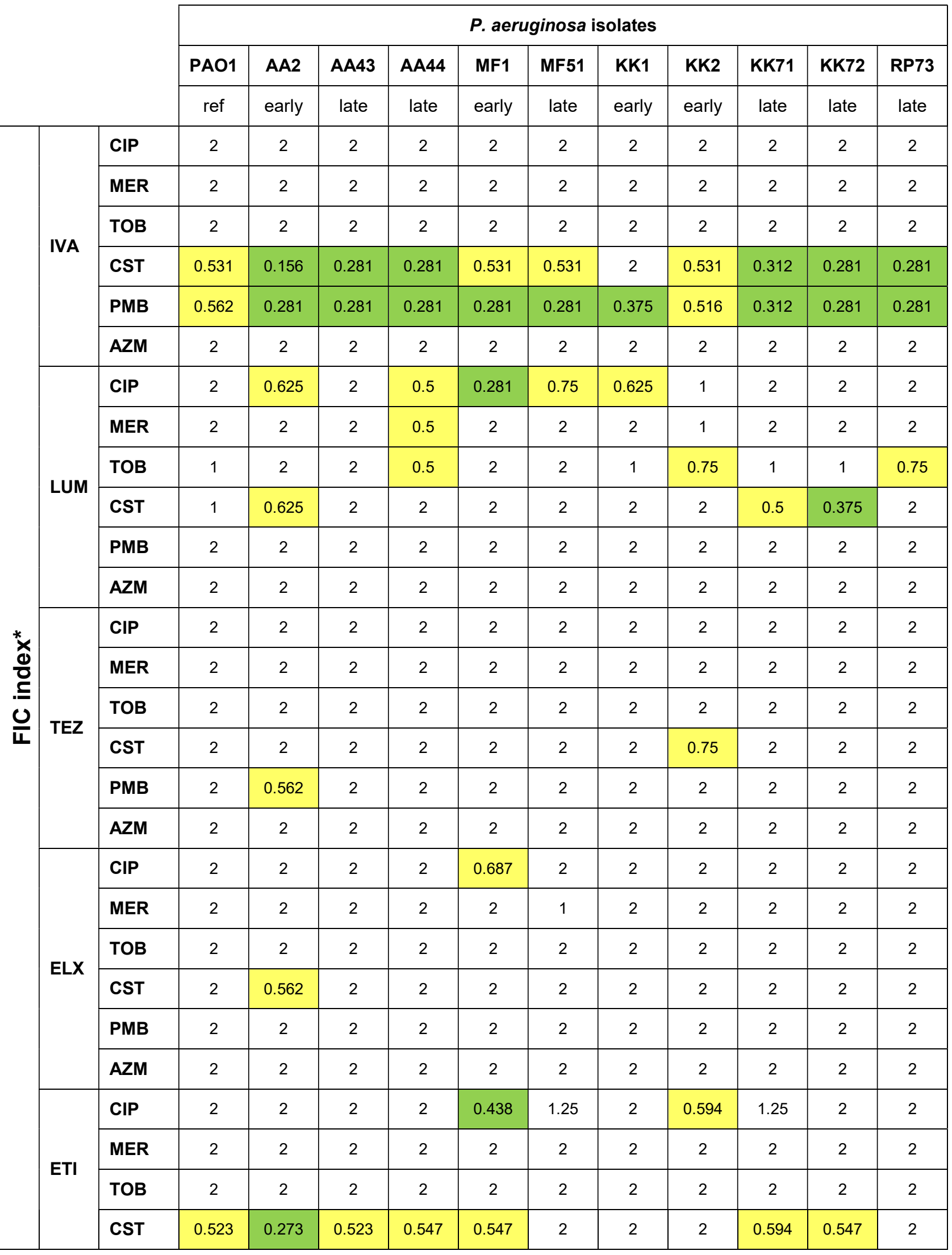




\begin{tabular}{|l|c|c|c|c|c|c|c|c|c|c|c|}
\hline PMB & 2 & 0.297 & 0.512 & 2 & 2 & 0.547 & 2 & 2 & 0.547 & 0.547 & 2 \\
\hline AZM & 2 & 2 & 2 & 2 & 2 & 2 & 2 & 2 & 2 & 2 & 2 \\
\hline
\end{tabular}

${ }^{*} \mathrm{FIC}$ index $<0.5$ indicates synergy (highlighted in green), FIC index $>0.50$ and $<1.0$ indicates additive effect (highlighted in yellow), FIC index $>1.0$ and $<4$ indicates indifference. Ref, reference strain; early, isolate collected at the early stage of chronic colonization; late, isolate collected after years of persistence, in the advanced stage of chronic colonization.

Table 4. Percentages of ivacaftor (IVA) and its metabolites (M1 and M6) as protein-bound and free active compounds in murine plasma and lungs. Blood was collected from C57BL/6NCrIBR male mice ( 8 to 10 weeks of age) and processed to obtain plasma. Lungs were excised, homogenized, and centrifuged, and the supernatants were collected. Protein binding was measured by the dialysis method with Rapid Equilibrium Dialysis inserts, with undiluted plasma or lung homogenate from 2-hr samples derived from non-infected untreated mice diluted 1:2 in PBS buffer spiked with a solution containing the three analytes against PBS buffer. Samples were analysed with ultra-performance liquid chromatography-tandem mass spectrometry in positive multiple reaction monitoring mode. Values are the means of three replicates.

\begin{tabular}{llccc}
\hline \multirow{2}{*}{ Sample } & & \multicolumn{3}{c}{ Protein binding (\%) } \\
\cline { 3 - 5 } & & IVA & M1 & M6 \\
\hline \multirow{2}{*}{ Plasma } & Bound fraction & 99.97 & 99.89 & 99.47 \\
& Free active fraction & 0.03 & 0.11 & 0.53 \\
\hline \multirow{2}{*}{ Lung } & Bound fraction & 99.74 & 97.17 & 80.3 \\
& Free active fraction & 0.26 & 2.83 & 19.7 \\
\hline
\end{tabular}

Table 5. Pharmacokinetic parameter estimates for ivacaftor (IVA) in plasma, epithelial lining fluid (ELF) and lungs from infected and non-infected mice. C57BL/6NCrIBR male mice (8 to 10 weeks of age) were infected with $1 \times 10^{6}$ colony forming units of $P$. aeruginosa PAO1 by intratracheal administration. A non-infected control group was also tested in parallel. Thirty minutes after infection, the mice were treated with $3 \mathrm{mg} / \mathrm{kg}$ IVA in $10 \%$ PEG $400,10 \%$ Tween 80 , and $80 \%$ saline by intraperitoneal administration. Mice were sacrificed at 10 min and 1, 2, 6 and $24 \mathrm{hrs}$ after IVA administration. Blood was collected and processed to obtain plasma. Bronchoalveolar lavage fluid (BALF) was collected and centrifuged, and the supernatant was used to quantify the IVA concentration. Lungs were excised, homogenized, and centrifuged, and the supernatants were used to quantify IVA concentrations. Plasma, lung homogenate and BALF were added to a Phree phospholipid removal plate (Phenomenex) with acetonitrile and $0.1 \%$ formic acid in order to eliminate phospholipids, decreasing the matrix effect. Eluates were analysed by the ultra-performance liquid chromatography-tandem mass spectrometry method with a linear gradient in multiple reaction monitoring positive mode. Calibration ranges were $0.5-750 \mathrm{ng} / \mathrm{ml}$ in plasma and BALF and 4-1000 $\mathrm{ng} / \mathrm{g}$ in the lung homogenate. Concentrations of IVA in ELF were determined using the ratio of the urea concentration in plasma to that in BALF. Concentration in ELF $=$ drug concentration in BALF $\times$ urea in plasma / urea in BALF. The data are the geometric means of values from 3-4 mice.

\begin{tabular}{|c|c|c|c|c|c|c|c|c|c|}
\hline \multirow[b]{2}{*}{ Sample } & \multirow[b]{2}{*}{$\begin{array}{l}\text { Mouse } \\
\text { group }\end{array}$} & \multicolumn{8}{|c|}{ Pharmacokinetic parameters } \\
\hline & & $\begin{array}{l}T_{\max } \\
\text { (hrs) }\end{array}$ & $\begin{array}{c}\mathrm{C}_{\max } \\
(\mathrm{ng} / \mathrm{ml})\end{array}$ & $\begin{array}{l}\mathrm{T}_{\text {last }} \\
\text { (hrs) }\end{array}$ & $\begin{array}{l}\mathrm{C}_{\text {last }} \\
\text { (hrs) }\end{array}$ & $\begin{array}{c}\mathrm{AUC}_{\text {last }} \\
(\mathrm{hrs} \\
\mathrm{ng} / \mathrm{ml})\end{array}$ & $\begin{array}{c}\mathrm{AUC}_{\mathbb{I N}} \\
\mathrm{F} \\
(\mathrm{hrs} \\
\mathrm{ng} / \mathrm{ml})\end{array}$ & $\begin{array}{l}\mathrm{T}_{1 / 2} \\
\text { (hrs) }\end{array}$ & $\begin{array}{l}\text { MRT } \\
\text { (hrs) }\end{array}$ \\
\hline \multirow{2}{*}{ Plasma } & $\begin{array}{c}\text { non- } \\
\text { infected }\end{array}$ & 1 & 2345 & 24 & 4.0 & 7482 & 7498 & 2.9 & 2.9 \\
\hline & infected & 1 & 968 & 24 & 8.6 & 9111 & 9152 & 3.3 & 4.7 \\
\hline
\end{tabular}




\begin{tabular}{|l|c|c|c|c|c|c|c|c|c|}
\hline \multirow{2}{*}{ ELF } & $\begin{array}{c}\text { non- } \\
\text { infected }\end{array}$ & 1 & 537 & 6 & 116.7 & 1932 & 2307 & 2.2 & 2.1 \\
\cline { 2 - 10 } & infected & 1 & 981 & 6 & 194.3 & 2590 & 3236 & 2.3 & 2.2 \\
\hline \multirow{2}{*}{ Lung } & $\begin{array}{c}\text { non- } \\
\text { infected }\end{array}$ & 1 & 249 & 6 & 68.2 & 946 & nd & nd & 2.2 \\
\cline { 2 - 11 } & infected & 1 & 364 & 24 & 4.4 & 4010 & 4033 & 3.7 & 5.2 \\
\hline
\end{tabular}

$T_{\max }$, time of maximum concentration; $\mathrm{C}_{\max }$, maximum concentration; $\mathrm{T}_{\text {last, }}$ time of last quantifiable concentration; $\mathrm{C}_{\text {last, }}$ last quantifiable concentration; $A \cup C_{\text {last }}, A U C$ to the last quantifiable concentration level; $A U C_{I N F}, A U C$ to infinity; $T_{1 / 2}$, half-life; MRT, mean residence time; nd, not determined.

Table 6. Penetration of ivacaftor (IVA) in epithelial lining fluid (ELF) and lungs. The $C_{\max }$ and AUC of plasma and lung homogenates were corrected for the free fraction according to the protein binding experiment $(0.03 \%$ for plasma and $0.26 \%$ for lung; Table 4$)$ to obtain the $\mathrm{C}_{\max }$ and AUC of unbound IVA ( $\mathrm{fC}_{\max }$ and $\mathrm{fAUC}$ ). Data are the geometric means of values from 3-4 mice.

\begin{tabular}{|c|c|c|c|c|c|c|c|c|c|c|}
\hline \multirow[b]{3}{*}{$\begin{array}{l}\text { Mouse } \\
\text { group }\end{array}$} & \multicolumn{6}{|c|}{ Values by sample type } & \multirow{2}{*}{\multicolumn{2}{|c|}{ Lung/plasma }} & \multirow{2}{*}{\multicolumn{2}{|c|}{ ELF/plasma }} \\
\hline & \multicolumn{2}{|c|}{ plasma } & \multicolumn{2}{|c|}{ lung } & \multicolumn{2}{|c|}{ ELF } & & & & \\
\hline & $\begin{array}{c}\mathrm{fC}_{\max } \\
(\mathrm{ng} / \mathrm{ml} \\
)\end{array}$ & $\begin{array}{c}\text { fAUC } \\
\text { (hrs } \\
\text { ng/ml) }\end{array}$ & $\begin{array}{c}\mathrm{fC}_{\max } \\
(\mathrm{ng} / \mathrm{ml} \\
)\end{array}$ & $\begin{array}{c}\text { fAUC } \\
\text { (hrs } \\
\mathrm{ng} / \mathrm{ml} \text { ) }\end{array}$ & $\begin{array}{c}\mathrm{C}_{\max } \\
(\mathrm{ng} / \mathrm{ml} \\
)\end{array}$ & $\begin{array}{c}\text { AUC } \\
\text { (hrs } \\
\mathrm{ng} / \mathrm{ml})\end{array}$ & $\mathrm{C}_{\max }^{\#}$ & $A \cup C^{\#}$ & $\mathrm{C}_{\max }^{\#}$ & $\mathrm{AUC}^{\#}$ \\
\hline $\begin{array}{l}\text { Non- } \\
\text { infected }\end{array}$ & 0.70 & 2.20 & 0.65 & 2.44 & 537 & 1910 & 0.93 & 1.11 & 767 & 868 \\
\hline Infected & 0.29 & 2.72 & 0.94 & 10.62 & 981 & 2587 & 3.24 & 3.90 & 3383 & 951 \\
\hline
\end{tabular}

\#Values for lung/plasma (ratio of lung free maximum concentration or lung exposure to free maximum concentration divided by those in plasma) and ELF/plasma (ratio of ELF maximum concentration or ELF exposure to maximum concentration divided by plasma free maximum concentration or plasma exposure to free maximum concentration); $\mathrm{fC}_{\max }$ and $\mathrm{fAUC}_{\mathrm{C}}$ refer to unbound IVA in the plasma and lung.

\section{Figure}

A

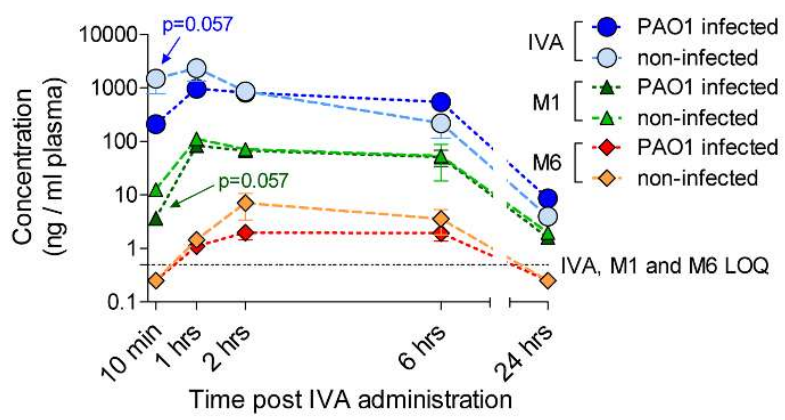

B

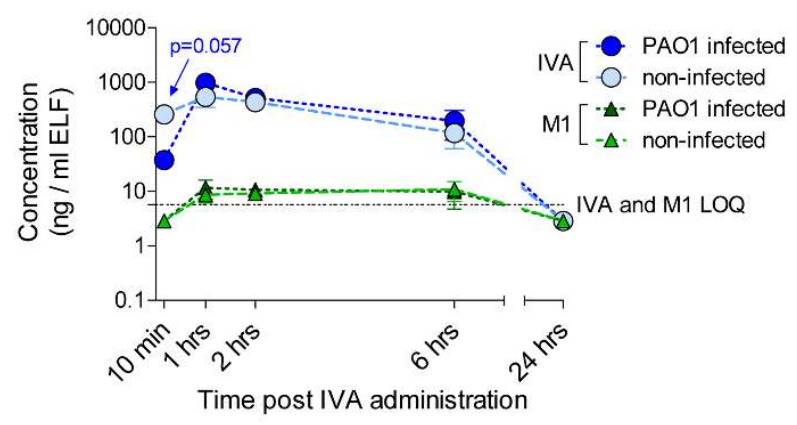

Figure 1. Ivacaftor (IVA), M1 and M6 concentrations in murine plasma and epithelial lining fluid (ELF). C57BL/6NCrIBR male mice (8 to 10 weeks of age) were infected with $1 \times 10^{6}$ colony forming units of $P$. aeruginosa PAO1 by intratracheal administration. A non-infected control group was also tested in parallel. Thirty minutes after infection, the mice were treated with $3 \mathrm{mg} / \mathrm{kg}$ IVA in $10 \%$ PEG $400,10 \%$ Tween 80 , and $80 \%$ saline by intraperitoneal administration. Mice were sacrificed at $10 \mathrm{~min}$ and 1, 2, 6 and $24 \mathrm{hrs}$ after IVA administration. Blood was collected and processed to obtain plasma. IVA, M1 and M6 concentrations in plasma were measured by highperformance liquid chromatography-tandem mass spectrometry (A). Bronchoalveolar lavage fluid (BALF) was collected and centrifuged, and the supernatant was used to quantify the IVA concentration. The volume of the ELF was determined by using the ratio of the urea concentration 
405 in the BALF to that in the plasma (B). The amounts of IVA and M1 were measured by high406 performance liquid chromatography-tandem mass spectrometry. Data (derived from 3-4 mice) are 407 represented as the mean values \pm standard errors of the means (SEMs). Limits of quantification 408 (LOQ) for IVA and its metabolites are indicated. A value corresponding to LOQ/2 was assigned to 409 undetectable samples at specific time-points (e.g. $10 \mathrm{~min}$ and/or $24 \mathrm{hrs}$ ). Statistical significance was 410 calculated by the Mann-Whitney test comparing the infected and non-infected mouse groups at each 411 time point. 


\section{Bibliography}

1. Gibson RL, Burns JL, Ramsey BW. Pathophysiology and management of pulmonary infections in cystic fibrosis. Am J Respir Crit Care Med 2003: 168(8): 918-951.

2. Camus L, Briaud P, Vandenesch F, Moreau K. How Bacterial Adaptation to Cystic Fibrosis Environment Shapes Interactions Between Pseudomonas aeruginosa and Staphylococcus aureus. Frontiers in microbiology 2021: 12: 617784.

3. Shteinberg M, Haq IJ, Polineni D, Davies JC. Cystic fibrosis. Lancet 2021: 397(10290): 21952211.

4. Ramsey BW, Davies J, McElvaney NG, Tullis E, Bell SC, Drevinek P, Griese M, McKone EF, Wainwright CE, Konstan MW, Moss R, Ratjen F, Sermet-Gaudelus I, Rowe SM, Dong Q, Rodriguez S, Yen K, Ordonez C, Elborn JS, Group VXS. A CFTR potentiator in patients with cystic fibrosis and the G551D mutation. N Engl J Med 2011: 365(18): 1663-1672.

5. Durmowicz AG, Witzmann KA, Rosebraugh CJ, Chowdhury BA. Change in sweat chloride as a clinical end point in cystic fibrosis clinical trials: the ivacaftor experience. Chest 2013: 143(1): 14-18.

6. Millar BC, McCaughan J, Rendall JC, Downey DG, Moore JE. Pseudomonas aeruginosa in cystic fibrosis patients with c.1652GA (G551D)-CFTR treated with ivacaftor-Changes in microbiological parameters. J Clin Pharm Ther 2018: 43(1): 92-100.

7. Wainwright CE, Elborn JS, Ramsey BW. Lumacaftor-Ivacaftor in Patients with Cystic Fibrosis Homozygous for Phe508del CFTR. N Engl J Med 2015: 373(18): 1783-1784.

8. Konstan MW, McKone EF, Moss RB, Marigowda G, Tian S, Waltz D, Huang X, Lubarsky B, Rubin J, Millar SJ, Pasta DJ, Mayer-Hamblett N, Goss CH, Morgan W, Sawicki GS. Assessment of safety and efficacy of long-term treatment with combination lumacaftor and ivacaftor therapy in patients with cystic fibrosis homozygous for the F508del-CFTR mutation (PROGRESS): a phase 3 , extension study. Lancet Respir Med 2017: 5(2): 107-118.

9. Rowe SM, Daines C, Ringshausen FC, Kerem E, Wilson J, Tullis E, Nair N, Simard C, Han L, Ingenito EP, McKee C, Lekstrom-Himes J, Davies JC. Tezacaftor-Ivacaftor in Residual-Function Heterozygotes with Cystic Fibrosis. N Engl J Med 2017: 377(21): 2024-2035.

10. Taylor-Cousar JL, Munck A, McKone EF, van der Ent CK, Moeller A, Simard C, Wang LT, Ingenito EP, McKee C, Lu Y, Lekstrom-Himes J, Elborn JS. Tezacaftor-Ivacaftor in Patients with Cystic Fibrosis Homozygous for Phe508del. N Engl J Med 2017: 377(21): 2013-2023.

11. Donaldson SH, Pilewski JM, Griese M, Cooke J, Viswanathan L, Tullis E, Davies JC, Lekstrom-Himes JA, Wang LT, Group VXS. Tezacaftor/lvacaftor in Subjects with Cystic Fibrosis and F508del/F508del-CFTR or F508del/G551D-CFTR. Am J Respir Crit Care Med 2018: 197(2): 214224.

12. Keating D, Marigowda G, Burr L, Daines C, Mall MA, McKone EF, Ramsey BW, Rowe SM, Sass LA, Tullis E, McKee CM, Moskowitz SM, Robertson S, Savage J, Simard C, Van Goor F, Waltz D, Xuan F, Young T, Taylor-Cousar JL. VX-445-Tezacaftor-Ivacaftor in Patients with Cystic Fibrosis and One or Two Phe508del Alleles. The New England journal of medicine 2018: 379(17): 16121620.

13. Middleton PG, Mall MA, Drevinek P, Lands LC, McKone EF, Polineni D, Ramsey BW, TaylorCousar JL, Tullis E, Vermeulen F, Marigowda G, McKee CM, Moskowitz SM, Nair N, Savage J, Simard C, Tian S, Waltz D, Xuan F, Rowe SM, Jain R. Elexacaftor-Tezacaftor-Ivacaftor for Cystic Fibrosis with a Single Phe508del Allele. The New England journal of medicine 2019: 381(19): 18091819.

14. Yi B, Dalpke AH, Boutin S. Changes in the Cystic Fibrosis Airway Microbiome in Response to CFTR Modulator Therapy. Frontiers in cellular and infection microbiology 2021: 11: 548613.

15. Volkova N, Moy K, Evans J, Campbell D, Tian S, Simard C, Higgins M, Konstan MW, Sawicki GS, Elbert A, Charman SC, Marshall BC, Bilton D. Disease progression in patients with cystic fibrosis treated with ivacaftor: Data from national US and UK registries. Journal of cystic fibrosis : official journal of the European Cystic Fibrosis Society 2020: 19(1): 68-79.

16. Hisert KB, Heltshe SL, Pope C, Jorth P, Wu X, Edwards RM, Radey M, Accurso FJ, Wolter DJ, Cooke G, Adam RJ, Carter S, Grogan B, Launspach JL, Donnelly SC, Gallagher CG, Bruce JE, Stoltz DA, Welsh MJ, Hoffman LR, McKone EF, Singh PK. Restoring Cystic Fibrosis 
bioRxiv preprint doi: https://doi.org/10.1101/2022.02.15.478594; this version posted February 15, 2022. The copyright holder for this preprint (which was not certified by peer review) is the author/funder. All rights reserved. No reuse allowed without permission.

Transmembrane Conductance Regulator Function Reduces Airway Bacteria and Inflammation in People with Cystic Fibrosis and Chronic Lung Infections. Am J Respir Crit Care Med 2017: 195(12): 1617-1628.

17. Durfey SL, Pipavath S, Li A, Vo AT, Ratjen A, Carter S, Morgan SJ, Radey MC, Grogan B, Salipante SJ, Welsh MJ, Stoltz DA, Goss CH, McKone EF, Singh PK. Combining Ivacaftor and Intensive Antibiotics Achieves Limited Clearance of Cystic Fibrosis Infections. MBio 2021: 12(6): e0314821.

18. Reznikov LR, Abou Alaiwa MH, Dohrn CL, Gansemer ND, Diekema DJ, Stoltz DA, Welsh MJ. Antibacterial properties of the CFTR potentiator ivacaftor. Journal of cystic fibrosis : official journal of the European Cystic Fibrosis Society 2014: 13(5): 515-519.

19. Payne JE, Dubois AV, Ingram RJ, Weldon S, Taggart CC, Elborn JS, Tunney MM. Activity of innate antimicrobial peptides and ivacaftor against clinical cystic fibrosis respiratory pathogens. Int $J$ Antimicrob Agents 2017: 50(3): 427-435.

20. Cho DY, Lim DJ, Mackey C, Skinner D, Zhang S, McCormick J, Woodworth BA. Ivacaftor, a Cystic Fibrosis Transmembrane Conductance Regulator Potentiator, Enhances Ciprofloxacin Activity Against Pseudomonas aeruginosa. American journal of rhinology \& allergy 2019: 33(2): 129136.

21. Schneider EK, Azad MA, Han ML, Tony Zhou Q, Wang J, Huang JX, Cooper MA, Doi Y, Baker MA, Bergen PJ, Muller MT, Li J, Velkov T. An "Unlikely" Pair: The Antimicrobial Synergy of Polymyxin B in Combination with the Cystic Fibrosis Transmembrane Conductance Regulator Drugs KALYDECO and ORKAMBI. ACS Infect Dis 2016: 2(7): 478-488.

22. Thakare R, Singh AK, Das S, Vasudevan N, Jachak GR, Reddy DS, Dasgupta A, Chopra S. Repurposing Ivacaftor for treatment of Staphylococcus aureus infections. International journal of antimicrobial agents 2017: 50(3): 389-392.

23. Allobawi R, Ghelani DP, Schneider-Futschik EK. Metabolomic Description of Ivacaftor Elevating Polymyxin B Mediated Antibacterial Activity in Cystic Fibrosis Pseudomonas aeruginosa. ACS Pharmacol Transl Sci 2020: 3(3): 433-443.

24. Rodvold KA, George JM, Yoo L. Penetration of anti-infective agents into pulmonary epithelial lining fluid: focus on antibacterial agents. Clinical pharmacokinetics 2011: 50(10): 637-664.

25. CfDEaR USFaDA. Ivacaftor NDA 202188, Clinical Inspection Summary, Access data. FDA:203188Orif1s000 2012.

26. Matthes E, Goepp J, Carlile GW, Luo Y, Dejgaard K, Billet A, Robert R, Thomas DY, Hanrahan JW. Low free drug concentration prevents inhibition of F508del CFTR functional expression by the potentiator VX-770 (ivacaftor). British journal of pharmacology 2016: 173(3): 459470.

27. Schneider EK, Reyes-Ortega F, Wilson JW, Kotsimbos T, Keating D, Li J, Velkov T. Development of HPLC and LC-MS/MS methods for the analysis of ivacaftor, its major metabolites and lumacaftor in plasma and sputum of cystic fibrosis patients treated with ORKAMBI or KALYDECO. Journal of chromatography B, Analytical technologies in the biomedical and life sciences 2016: 1038: 57-62.

28. Cigana C, Bernardini F, Facchini M, Alcala-Franco B, Riva C, De Fino I, Rossi A, Ranucci S, Misson P, Chevalier E, Brodmann M, Schmitt M, Wach A, Dale GE, Obrecht D, Bragonzi A. Efficacy of the Novel Antibiotic POL7001 in Preclinical Models of Pseudomonas aeruginosa Pneumonia. Antimicrobial agents and chemotherapy 2016: 60(8): 4991-5000.

29. Hirschhausen N, Block D, Bianconi I, Bragonzi A, Birtel J, Lee JC, Dubbers A, Kuster P, Kahl $\mathrm{J}$, Peters G, Kahl BC. Extended Staphylococcus aureus persistence in cystic fibrosis is associated with bacterial adaptation. International journal of medical microbiology : IJMM 2013: 303(8): 685692.

30. Lore NI, Cigana C, De Fino I, Riva C, Juhas M, Schwager S, Eberl L, Bragonzi A. Cystic fibrosis-niche adaptation of Pseudomonas aeruginosa reduces virulence in multiple infection hosts. PLoS One 2012: 7(4): e35648.

31. Bianconi I, Jeukens J, Freschi L, Alcala-Franco B, Facchini M, Boyle B, Molinaro A, KukavicaIbrulj I, Tummler B, Levesque RC, Bragonzi A. Comparative genomics and biological characterization of sequential Pseudomonas aeruginosa isolates from persistent airways infection. BMC Genomics 2015: 16: 1105. 
32. CLSI. CLSI M100-S24. Performance standards for antimicrobial susceptibility testing; 24th informational supplement. . Clinical and Laboratory Standards Institute, Wayne, Pa 2014.

33. Sousa MGC, Xavier PD, Cantuaria APC, Porcino RA, Almeida JA, Franco OL, Rezende TMB. Host defense peptide IDR-1002 associated with ciprofloxacin as a new antimicrobial and immunomodulatory strategy for dental pulp revascularization therapy. Microbial pathogenesis 2021: 152: 104634.

34. Percie du Sert N, Hurst V, Ahluwalia A, Alam S, Avey MT, Baker M, Browne WJ, Clark A, Cuthill IC, Dirnagl U, Emerson M, Garner P, Holgate ST, Howells DW, Karp NA, Lazic SE, Lidster K, MacCallum CJ, Macleod M, Pearl EJ, Petersen OH, Rawle F, Reynolds P, Rooney K, Sena ES, Silberberg SD, Steckler T, Wurbel H. The ARRIVE guidelines 2.0: Updated guidelines for reporting animal research. PLoS biology 2020: 18(7): e3000410.

35. Cigana C, Ranucci S, Rossi A, De Fino I, Melessike M, Bragonzi A. Antibiotic efficacy varies based on the infection model and treatment regimen for Pseudomonas aeruginosa. The European respiratory journal 2020: 55(3).

36. Melchers MJ, Teague J, Warn P, Hansen J, Bernardini F, Wach A, Obrecht D, Dale GE, Mouton JW. Pharmacokinetics and Pharmacodynamics of Murepavadin in Neutropenic Mouse Models. Antimicrobial agents and chemotherapy 2019: 63(3).

37. Hoy SM. Elexacaftor/lvacaftor/Tezacaftor: First Approval. Drugs 2019: 79(18): 2001-2007.

38. Harun SN, Wainwright C, Klein K, Hennig S. A systematic review of studies examining the rate of lung function decline in patients with cystic fibrosis. Paediatr Respir Rev 2016: 20: 55-66.

39. Condren ME, Bradshaw MD. Ivacaftor: a novel gene-based therapeutic approach for cystic fibrosis. J Pediatr Pharmacol Ther 2013: 18(1): 8-13.

40. Pharmaceuticals V. Ivacaftor. https://pivrtxcom/files/uspi_ivacaftorpdf.

41. Chen J, Ahmed MU, Zhu C, Yu S, Pan W, Velkov T, Li J, Tony Zhou Q. In vitro evaluation of drug delivery behavior for inhalable amorphous nanoparticle formulations in a human lung epithelial cell model. International journal of pharmaceutics 2021: 596: 120211. 